\title{
Active control of sound with a one-dimensional secondary source array
}

Masaki Hasebe

Department of Sanitary and Environmental Engineering, Faculty of Engineering, Hokkaido University, Kita-13, Nishi-8, Kita-ku, Sapporo, 060 Japan

(Received 25 March 1996)

Keywords: Active noise control, JMC method, 3-Dimensional space, Tripole PACS number: 43. 50. Ki

\section{Introduction}

The JMC (Jessel-Mangiante-Canévet) method, ${ }^{1)}$ based on Huygens' principle, has been proposed for controlling a free field sound. However, a continuous source layer must be used to ensure perfect control of a free field sound, which is still technologically impossible at this stage. A network of discrete secondary sound sources is currently used to approximate a continuous source layer. Each secondary source, which is made up of a monopole/dipole combination (tripole), is required to absorb the incident sound wave.

Several studies have been conducted on the applicability of a network of secondary sources. ${ }^{2,3)}$ In addition, numerical simulation ${ }^{4)}$ has shown that a network of secondary sources reduces the sound pressure level distribution over 3-dimensional space. A large number of secondary sources was found to be necessary to control the sound field. The maximum number of the tripole sound sources which have been realized in an experimental study is only three. ${ }^{2)}$ This is a reason why the method is inconvenient for practical use. Thus, the number of secondary sources must be reduced to make the JMC method practical. This paper presents a simple method to approximate the network surface of secondary sources by a source array, which can greatly reduce the number of secondary sources. The results of numerical simulation are also shown.

\section{A calculation model}

Mangiante $^{4)}$ has shown a typical result of a numerical simulation with distributed discrete secondary sources on a surface of a prolate spheroid (major axis $2 a=100$ $\mathrm{m}$, minor axis $2 b=10 \mathrm{~m}$; see Fig. 1). The primary source was situated far $(4000 \mathrm{~m})$ from the center of the spheroid, thus reducing the sound pressure level in the inner space of the spheroid. In this study, the calculation model which was used by Mangiante was modified, and the results were compared. The target field $P_{\mathrm{T}}$, which is emitted from distributed discrete secondary sources on a surface, is as follows :

$$
\begin{aligned}
P_{\mathrm{T}}= & -\frac{1}{4 \pi} \sum_{m} \sum_{n} A\left(j k+\frac{1}{r_{0 m n}}\right) \frac{e^{-j k\left(r_{0} n+R m n\right)}}{r_{0 m n} R_{m n}} \cos \theta_{m n} \Delta \Sigma_{0 m n} \\
& -\frac{1}{4 \pi} \sum_{m} \sum_{n} A\left(j k+\frac{1}{R_{m n}}\right) \frac{e^{-j k\left(r_{0} n+R_{m n}\right)}}{r_{0 m n} R_{m n}} \cos \xi_{m n} \Delta \Sigma_{0 m n}
\end{aligned}
$$

$\Delta \Sigma_{0 m n}$ is the unit area around the secondary source point $(m, n)$ on the surface of a prolate spheroid. If we assume that the sound field from a primary sound source is only varying slowly around the prolate spheroid, we can approximate the summation over the vertical space to a point on a $x-y$ plane, i.e., the secondary sources are distributed only on the edge of ellipse. Under the condition $r_{0 m n} \gg j k, R_{m n} \gg j k$, and using the method of stationary phase, ${ }^{5)}$ the approximated target field can be shown as

$$
\begin{aligned}
P_{\mathrm{T}}= & \frac{k^{1 / 2} e^{-i \pi / 4}}{8 \sqrt{2} \pi^{3 / 2}}\left(\sum_{m} \frac{e^{-j k\left(r_{0}+R_{m}\right)}}{\sqrt{\left(r_{0 m} R_{m}\left(r_{0 m}+R_{m}\right)\right)}} \cos \theta_{m} \Delta d_{0 m}\right. \\
& \left.-\sum_{m} \frac{e^{-j k\left(r_{0 m}+R_{m}\right)}}{\sqrt{\left(r_{0 m} R_{m}\left(r_{0 m}+R_{m}\right)\right)}} \cos \xi_{m} \Delta d_{0 m}\right)
\end{aligned}
$$

Using the condition that the primary source is far from the spheroid, i.e. $r_{0 m n} \gg R_{m n}$, the approximated target field can be shown as

$$
\begin{aligned}
P_{\mathrm{T}}= & \frac{k^{1 / 2} e^{-i \pi / 4}}{8 \sqrt{2} \pi^{3 / 2}}\left(\sum_{m} \frac{e^{-j k\left(r_{0}+R_{m}\right)}}{\sqrt{\left(r_{0 m}^{2} R_{m}\right)}} \cos \theta_{m} \Delta d_{0 m}\right. \\
& \left.-\sum_{m} \frac{e^{-j k\left(r_{0 m}+R_{m}\right)}}{\sqrt{\left({r_{0 m}}^{2} R_{m}\right)}} \cos \xi_{m} \Delta d_{0 m}\right)
\end{aligned}
$$

\section{Numerical simulation and results}

Using equation (1) for the secondary sources on the prolate spheroid, and equation (3) for the secondary sources on the edge of ellipse, numerical calculation was carried out at the points along the major axis of the prolate spheroid, for the case of sound in air and frequency of $50 \mathrm{~Hz}$. The number of secondary sources distributed on the prolate spheroid was 4287 and the number of secondary sources on the edge of ellipse in the $x-y$ plane was 264 .

The results are shown in Fig. 2 for the case of equation (1) and in Fig. 3 for the case of equation (3). Figure 2 shows that the attenuations are not greatly dependent on the height $z$ of the calculated points. However, in Fig. 3, the attenuations are dependent on the height of the calculated points. For the practical purposes, this shortcoming is not so important for the case, for example, where we need calm flat space centered around our ears. Moreover, it is preferable that the number of secondary sources are much smaller than that of the original case.

Equation (3) determines the sound field which 


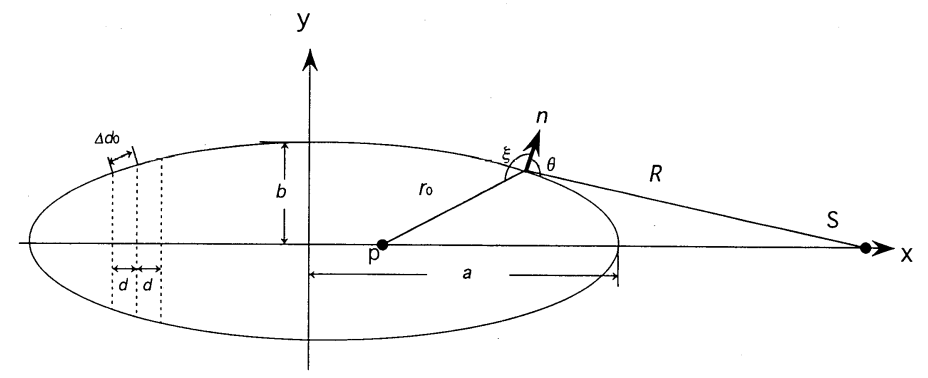

Fig. 1 Secondary sources distributed on a prolate spheroid. This figure shows a cross section in the $x-y$ plane.

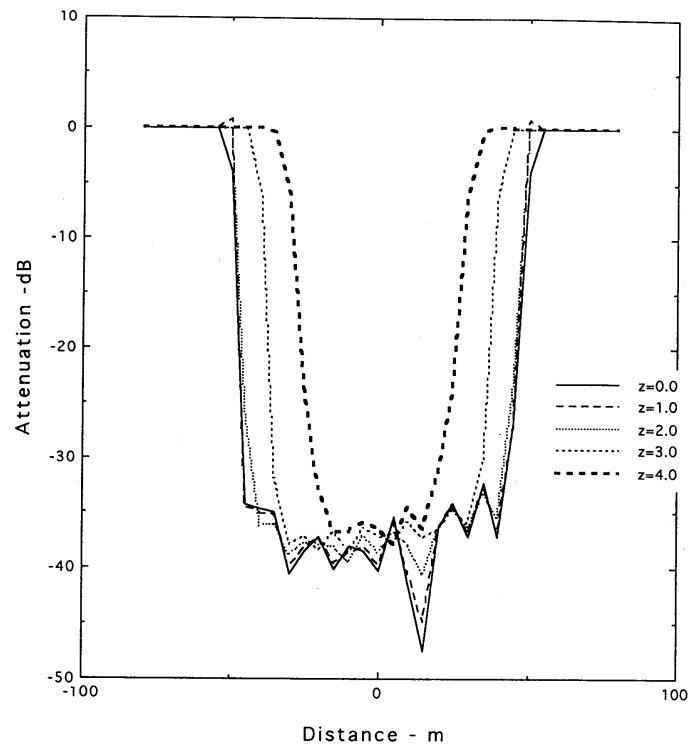

Fig. 2 Sound pressure level attenuation by the distribution on the prolate spheroid as a function of the height $z$ on the axis perpendicular to the $x-y$ plane.

should be emitted from the secondary source on the edge of the ellipse. The sound field should have the tendency of a line source, i.e., cylindrical waveform $\sim \exp \left(i k r_{0 m}\right) /\left(i k r_{0 m}\right)^{1 / 2}$ with an angular dependence of $\left(\cos \theta_{m}-\cos \xi_{m}\right)$. These suggest the characteristics of the secondary source which should be realized in a practical case. The angular dependence and characteristics of the line source can be realized using a conventional speaker unit with a limited frequency range.

\section{Acknowledgement}

This study was partially supported by a Grant-in-Aid for Scientific Research (No. 06650599) by the Ministry of Education, Science and Culture of Japan.

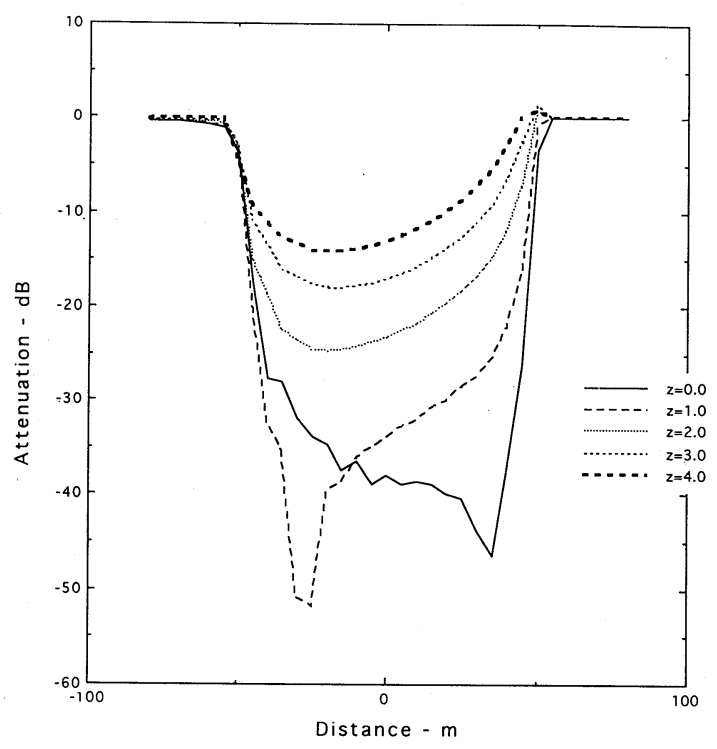

Fig. 3 Sound pressure level attenuation by the distribution on the edge of an ellipse as a function of the height $z$ on the axis perpendicular to the $x-y$ plane.

\section{References}

1) M. J. M. Jessel and G. Mangiante, "Active sound absorbers in air duct," J. Sound Vib. 23, 383-390 (1992).

2) M. Hasebe and M. Kaida, "Study on active noise attenuation in three-dimensional sound field," Proc. Inter Noise 93 (Leuven), 773-775 (1993).

3) G. Mangiante and A. Roure, "Autodirective sources for 3D active noise control," Proc. Inter Noise 94 (Yokohama), 773-775 (1993).

4) G. Mangiante, "The JMC method for 3D active sound absorption: A numerical simulation," Noise Control Eng. 41, 1293-1298 (1994).

5) E. Skudrzyk, The Foundations of Acoustics (Springer-Verlag, Wien, 1971). 